\title{
Robust FDI for a Class of Nonlinear Networked Systems with ROQs
}

\author{
An-quan Sun, Lin Zhang, Wen-feng Wang, Jun Wang, Tian-lin Niu, and Jia-li Zhang
}

Air and Missile Defense College, Air Force Engineering University, Xian 710051, China

Correspondence should be addressed to Wen-feng Wang; wwf981612@163.com

Received 9 September 2014; Accepted 3 November 2014; Published 20 November 2014

Academic Editor: Zidong Wang

Copyright (C) 2014 An-quan Sun et al. This is an open access article distributed under the Creative Commons Attribution License, which permits unrestricted use, distribution, and reproduction in any medium, provided the original work is properly cited.

\begin{abstract}
This paper considers the robust fault detection and isolation (FDI) problem for a class of nonlinear networked systems (NSs) with randomly occurring quantisations (ROQs). After vector augmentation, Lyapunov function is introduced to ensure the asymptotically mean-square stability of fault detection system. By transforming the quantisation effects into sector-bounded parameter uncertainties, sufficient conditions ensuring the existence of fault detection filter are proposed, which can reduce the difference between output residuals and fault signals as small as possible under $H_{\infty}$ framework. Finally, an example linearized from a vehicle system is introduced to show the efficiency of the proposed fault detection filter.
\end{abstract}

\section{Introduction}

With the wide use of computer network and the decrease of networked technique's cost, structures of control systems are changed. The wires of traditional control systems were replaced by computer network reserved for special or public use; thus information can be transmitted via controllers, sensors, actuators, and other system units [1]. This type of control systems is used or will be used not only in large-scale distributed systems (such as large-scale industrial control systems), but also in centralized small-scale local area systems (such as space vehicles, airliners, ships, high-performance motors, etc.) $[2,3]$. For this type of control systems, information like detection, control, coordinate, instruction, and so forth is transmitted via public network. While functions like estimation, control, diagnosis, and so forth can be conducted among different network nodes distributively. Control systems with at least one loop or several loops to make up closed loop by networks are called networked systems (NSs). Compared to traditional control systems, NSs have less wires, which make them convenient to install and service. In this situation, NSs can reduce systems' weight and size, and they are also convenient to extend. As well as the advantages brought by NSs through sharing resources on network, new challenges and opportunities were brought to its systems and control theories, such as data transmission delay, data dropout, and immanent quantisation problem. During recent decades, many famous journals about automatic control have published special issues on NSs [4-7]. Specifically in 2003, report on the development of automatic control made by Astrom and some famous scholars attracted unprecedented attention to NSs, also plenty of research results published [8]. However, compared to the comprehensive researches on NSs' stability [9-11], control [12, 13], and filtration [14-16], researches on NSs' fault detection seem to be less.

Fault detection is a technique to determine whether some characteristics or parameters of the system arouse larger deviation, which is unacceptable. It is a key technique which can improve system's security and reliability, and developed rapidly in the past thirty years. Fault detection belongs to the fault determination part in fault diagnosis procedure. A typical fault diagnosis procedure includes residual generation, residual evaluation, separation strategy, fault estimate, and performance evaluation. Firstly, generate residual signals when fault occurs and the signals are sensitive to the fault and robust to unknown input and system parameters uncertainty $[16,17]$. The most ideal condition to reach the above demand is to make residuals decoupled with unknown inputs, related only to faults. But the decoupling conditions are usually severe and most systems are unable to reach. In the latest 
years, a generally adopted approach is transforming the residual generation problem into optimization problem. Design a fault detection filter (residual generator), which makes the transfer function's $H_{\infty}$ bound norm from disturbance to residual as small as possible but the transfer function's $H_{\infty}$ bound norm from (or other bound norms) fault to residual as large as possible. Some existing researches demonstrate that there exists a unified solving method among different optimal indices $[18,19]$. After the residuals are generated, select a suitable residual evaluation function and a threshold value. Residual evaluation functions and threshold values can be different in form, but usually we take residual evaluation function over than threshold value as the judgment of fault alarm. Here is the description of fault detection problem; this is what we care about in this paper. After fault detection is done, faults are located with suitable separation strategy; level of the faults is estimated by kinds of approaches; superiority of the diagnosis algorithm is evaluated. This is the complete fault diagnosis procedure.

Most of the existing fault detection theories are set up based on traditional control systems, whose data transmissions are without delay or with ideal data dropout among system components. The appearance of NSs brings new challenges to traditional fault detection theories and out of this the main problem is that the signals transmitted via network became imperfect, including delay, data dropout, quantisation effect, and other signal changes not expected during the design. Compared to traditional control systems, the difficulty of NSs' fault detection mainly depends on the imperfection of observation signals. By now, most of the existing research results of NSs' diagnosis detection results focus on dealing with network induced delay, and few of them focus on data dropout, multipacket transmission, or signal quantisation [20-23]. In [24-27], the authors studied system's stability and control problem with information coder. In [28], Fu and Xie discussed the logarithmic quantiser and designed the corresponding state feedback controller. Yang et al. in [29] designed a networked filter based on logarithmic quantiser. In [30], Li et al. studied NSs' fault diagnosis problem, whose residual errors have quantisation errors, and then designed fault diagnosis filters, respectively, based on static and dynamic coders. But so far, to the best of the authors' knowledge, there are very few related results in the literature that focus on quantisation problems for NSs with randomly occurring quantisations (ROQs). This inspires our current research.

In this paper, the robust fault detection and isolation (FDI) problem is investigated for a class of nonlinear NSs with ROQs. A time-variant system with unknown disturbances and nonlinear terms is studied. The parameters of the system are time-variant and satisfy norm-bounded condition, and all the quantisers are assumed to be in the logarithmic type with different quantisation laws. On the other hand, Bernoulli distributed stochastic sequence is used to decide which quantiser is to be used at the moment. After vector augmentation, the fault detection problem is transformed into robust $H_{\infty}$ filter problem: design a robust fault detection filter for all allowable time-variant parameters, external disturbance, and possible quantisations; reduce the difference between fault detection filter's output and fault signal as small as possible under $H_{\infty}$ framework. The Lyapunov function is introduced to ensure the asymptotically mean-square stability of fault detection system and satisfies the corresponding $H_{\infty}$ disturbance restraint conditions. A sufficient condition is presented to ensure the existence of robust fault detection filter in the form of Linear Matrix Inequality (LMI), as well as the calculation of fault detection filter parameters. An example linearized from a vehicle system is used to show the efficiency of the proposed method.

Notations. $\mathbb{R}^{n}$ is the $n$-dimensional Euclidean space. $l_{2}[0, \infty)$ is the space of all square-summable vector functions over $[0, \infty) . \delta(x, y)$ is the Kronecker delta function, for two integers $x$ and $y$, if $x=y, \delta(x, y)=1$; otherwise, $\delta(x, y)=0$. $\operatorname{Prob}\left\{\tau_{k}=i\right\}$ means the probability of stochastic event $\tau_{k}=i$. $I$ stands for unit matrix. $\mathbb{E}\{x\}$ is the mathematical expectation of a stochastic variable $x .\left|x_{k}\right|$ is the Euclidean norm vector of $x_{k} ;\|x\|=\left(\sum_{k=0}^{\infty}\left|x_{k}\right|^{2}\right)^{1 / 2}$ is the $l_{2}$ norm of $\left\{x_{k}\right\}$. In symmetric matrices, “*” represents a term that is induced by symmetry, and $\operatorname{diag}\{\cdots\}$ stands for a block-diagonal matrix.

\section{Problem Formulation}

Consider a class of nonlinear NSs with ROQs:

$$
\begin{gathered}
x_{k+1}=A_{k} x_{k}+E_{k} g\left(x_{k}\right)+B_{k} w_{k}+M_{k} f_{k} \\
y_{k}=C x_{k}+D w_{k},
\end{gathered}
$$

where $x_{k} \in \mathbb{R}^{n}$ is the system state vector; $w_{k} \in l_{2}[0, \infty) \subset$ $\mathbb{R}^{n_{w}}$ is the external disturbance; $f_{k} \in \mathbb{R}^{n_{f}}$ is the fault signal. $A_{k}, E_{k}, B_{k}$, and $M_{k}$ are time-variant matrices with suitable dimensions, and $A_{k}=A+\Delta A_{k}, E_{k}=E+\Delta E_{k}, B_{k}=B+$ $\Delta B_{k}$, and $M_{k}=M+\Delta M_{k}$, where $A, E, B$, and $M$ are known constant matrices. $\Delta A_{k}, \Delta E_{k}, \Delta B_{k}$, and $\Delta M_{k}$ are unknown matrices with sector-bounded conditions given by

$$
\left[\begin{array}{llll}
\Delta A_{k} & \Delta E_{k} & \Delta B_{k} & \Delta M_{k}
\end{array}\right]=N F_{k}\left[\begin{array}{llll}
H_{1} & H_{2} & H_{3} & H_{4}
\end{array}\right],
$$

where $\mathrm{N}, \mathrm{H}_{1}, \mathrm{H}_{2}, \mathrm{H}_{3}$, and $H_{4}$ are known matrices. $F_{k}$ satisfies $F_{k} F_{k}^{T} \leq I . y_{k}$ is the measurement signal under ideal transmission conditions; $C$ and $D$ are known constant matrices.

$g\left(x_{k}\right)$ is nonlinear vector function with sector restricted conditions described as [31]

$$
\left[g\left(x_{k}\right)-T_{1} x_{k}\right]^{T}\left[g\left(x_{k}\right)-T_{2} x_{k}\right] \leq 0, \quad \forall x_{k} \in \mathbb{R}^{n_{x}},
$$

where $T_{1}$ and $T_{2}$ are known constant matrices and $T=T_{1}-T_{2}$ is symmetric and positive definite matrix.

Considering the quantisation issue of NCs, in this paper, we model the observing function as

$$
\tilde{y}_{k}=\sum_{i=1}^{N} \delta\left(\tau_{k}, i\right) g_{i}\left(y_{k}\right),
$$

where $\tau_{k}$ is a Bernoulli distributed stochastic variable with a known distribution law $\operatorname{Prob}\left\{\tau_{k}=i\right\}=\mathbb{E}\left\{\delta\left(\tau_{k}, i\right)\right\}=p_{i}$, $\sum_{i=1}^{N} p_{i} \leq 1 . \tilde{y}_{k}$ is the measurement signal with quantisation effect. 
For each $i(1 \leq i \leq N), g_{i}(\cdot)$ is a time-invariant logarithmic quantiser, which can be described by

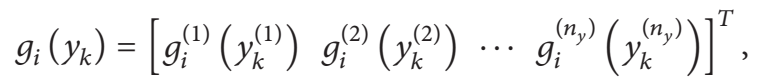

where $g_{i}^{(j)}(\cdot)\left(1 \leq j \leq n_{y}\right)$ is a scalar quantisation function with the quantisation levels described by

$$
\begin{array}{r}
\mathscr{u}_{i}^{(j)}=\left\{ \pm u_{i m}^{(j)} \mid u_{i m}^{(j)}=\left(\rho_{i}^{(j)}\right)^{m} u_{i 0}^{(j)}, m=0, \pm 1, \pm 2, \ldots\right\} \cup\{0\}, \\
0<\rho_{i}^{(j)}<1, \quad u_{i 0}^{(j)}>0 .
\end{array}
$$

Each of the quantisation levels corresponds to a segment; the data in one segment has the same quantisation output after being quantized. The logarithmic quantisers are described as

$$
g_{i}^{(j)}\left(y_{k}^{(j)}\right)= \begin{cases}u_{i m}^{(j)}, & \frac{1}{1+\delta_{i}^{(j)}} u_{i m}^{(j)}<y_{k}^{(j)} \leq \frac{1}{1-\delta_{i}^{(j)}} u_{i m}^{(j)} \\ 0, & y_{k}^{(j)}=0 \\ -g_{j}\left(-y_{k}^{(j)}\right), & y_{k}^{(j)}<0,\end{cases}
$$

where $\delta_{i}^{(j)}=\left(1-\rho_{i}^{(j)}\right) /\left(1+\rho_{i}^{(j)}\right)$. Then we can obtain $\delta_{i}^{(j)}\left(y_{k}^{(j)}\right)=\left(1+\Delta_{i k}^{(j)}\right) y_{k}^{(j)}$, where $\left|\Delta_{i k}^{(j)}\right| \leq \delta_{i}^{(j)}$. By defining $\Delta_{i k}=$ $\operatorname{diag}\left\{\Delta_{i k}^{(1)}, \ldots, \Delta_{i k}^{\left(n_{y}\right)}\right\}, \Delta_{i}=\operatorname{diag}\left\{\delta_{i}^{(1)}, \ldots, \delta_{i}^{\left(n_{y}\right)}\right\}$, and $F_{i k}=$ $\Delta_{i k} \Delta_{i}^{-1}$, we can obtain an unknown real-valued time-variant matrix satisfying $F_{i k} F_{i k}^{T} \leq I$. Furthermore, the measurements with quantisation effects are given by

$$
\tilde{y}_{k}=\sum_{i=1}^{N} \delta\left(\tau_{k}, i\right)\left(I+F_{i k} \Delta_{i}\right) y_{k} .
$$

Without loss of generality, the subscript $i$ of $F_{i k}$ can be ignored after vector augmentation and rewrite (8) as

$$
\begin{aligned}
\tilde{y}_{k} & =\sum_{i=1}^{N} \delta\left(\tau_{k}, i\right)\left(I+F_{k} \Delta_{i}\right) y_{k} \\
& =\sum_{i=1}^{N} \delta\left(\tau_{k}, i\right)\left(I+F_{k} \Delta_{i}\right) C x_{k}+\sum_{i=1}^{N} \delta\left(\tau_{k}, i\right)\left(I+F_{k} \Delta_{i}\right) D w_{k} .
\end{aligned}
$$

Consider a full-order filter described as

$$
\begin{gathered}
\widehat{x}_{k+1}=G \widehat{x}_{k}+K \tilde{y}_{k} \\
r_{k}=L \widehat{x}_{k},
\end{gathered}
$$

where $\widehat{x}_{k}$ is the state vector of the filter; $r_{k}$ is output residual; $G, K$, and $L$ are the parameters to be determined.
By defining $\eta_{k}=\left[\begin{array}{ll}x_{k}^{T} & \widehat{x}_{k}^{T}\end{array}\right]^{T}, v_{k}=\left[\begin{array}{ll}w_{k}^{T} & \widehat{w}_{k}^{T}\end{array}\right]^{T}$, and $\widetilde{r}_{k}=r_{k}-$ $f_{k}$, the augmented model is given by

$$
\begin{gathered}
\eta_{k+1}=\mathscr{A}_{k} \eta_{k}+\sum_{i=1}^{N}\left[\delta\left(\tau_{k}, i\right)-p_{i}\right] \mathscr{A}_{0} \eta_{k}+\mathscr{E}_{k} g\left(Z \eta_{k}\right)+\mathscr{B}_{k} v_{k} \\
\tilde{r}_{k}=r_{k}-f_{k}=\mathscr{C} \eta_{k}+\mathscr{D} v_{k},
\end{gathered}
$$

where

$$
\begin{aligned}
\mathscr{A}_{k}= & {\left[\begin{array}{cc}
A & 0 \\
\sum_{i=1}^{N} p_{i} K\left(I+F_{k} \Delta_{i}\right) C & G
\end{array}\right] } \\
& +\left[\begin{array}{c}
N \\
0
\end{array}\right] F_{k}\left[\begin{array}{ll}
H_{1} & 0
\end{array}\right]:=\mathscr{A}+\mathcal{N} F_{k} \mathscr{H}_{1}, \\
\mathscr{E}_{k}= & {\left[\begin{array}{c}
E \\
0
\end{array}\right]+\left[\begin{array}{c}
N \\
0
\end{array}\right] F_{k} H_{2}:=\mathscr{E}+\mathcal{N} F_{k} H_{2}, } \\
\mathscr{B}_{k}= & {\left[\begin{array}{cc}
\sum_{i=1}^{N} \delta\left(\tau_{k}, i\right) K\left(I+F_{k} \Delta_{i}\right) D & 0
\end{array}\right] } \\
& +\left[\begin{array}{c}
N \\
0
\end{array}\right] F_{k}\left[\begin{array}{cc}
H_{3} & H_{4}
\end{array}\right]:=\mathscr{B}+\mathscr{N} F_{k} \mathscr{H}_{3}, \\
\mathscr{A}_{0}= & {\left[\begin{array}{cc}
K\left(I+F_{k} \Delta_{i}\right) C & 0
\end{array}\right], } \\
\mathscr{C}_{=} & {\left[\begin{array}{ccc}
0 & L
\end{array}\right], \quad \mathscr{D}_{0}\left[\begin{array}{ll}
0 & -I
\end{array}\right], \quad Z=\left[\begin{array}{ll}
I & 0
\end{array}\right] . }
\end{aligned}
$$

After the above augmentation, the fault detection filter problem can be formulated as design robust $H_{\infty}$ filter.

The design of fault detection filter can be converted into determining a series of filter parameters, $G, K$, and $L$, to make the system satisfy two conditions:

(1) under zero input condition, system (11) is asymptotically mean-square stable;

(2) under zero initial condition, for all possible nonzero inputs $v_{k}$, the output $\widetilde{r}_{k}$ of (11) satisfies

$$
\mathbb{E}\left\{\|\widetilde{r}\|^{2}\right\}<\gamma^{2}\|\nu\|^{2},
$$

where $\gamma>0$ is a prescribed $H_{\infty}$ disturbance scalar.

\section{Main Results}

\subsection{Robust Fault Detection Filter Analysis}

Theorem 1. For a given scalar $\gamma>0$, if there exist scalar $\delta$ and symmetric and positive definite matrix $P=P^{T}>0$ making the following LMI holds:

$$
\Phi:=\left[\begin{array}{ccc}
\mathscr{A}_{k}^{T} P \mathscr{A}_{k}+\sum_{i=1}^{N} p_{i}\left(1-p_{i}\right) \mathscr{A}_{0}^{T} P \mathscr{A}_{0}-P-\delta \overline{\mathscr{T}}_{1}+\mathscr{C}^{T} \mathscr{C}_{k} & \mathscr{A}_{k}^{T} P \mathscr{E}_{k}-\delta \overline{\mathscr{T}}_{2} & \mathscr{A}_{k}^{T} P \mathscr{B}_{k}+\mathscr{C}^{T} \mathscr{D} \\
* & \mathscr{E}_{k}^{T} P \mathscr{E}_{k}-\delta I & \mathscr{E}_{k}^{T} P \mathscr{B}_{k} \\
* & * & \mathscr{B}_{k}^{T} P \mathscr{B}_{k}-\gamma^{2} I+\mathscr{D}^{T} \mathscr{D}
\end{array}\right]<0
$$


where $\mathscr{T}_{1}=\left(T_{1}^{T} T_{2}+T_{2}^{T} T_{1}\right) / 2$ and $\mathscr{T}_{2}=-\left(T_{1}^{T}+T_{2}^{T}\right) / 2$, then system (11) satisfies the aforementioned two conditions.

Proof. Consider the following Lyapunov function:

$$
V_{k}=\eta_{k}^{T} P \eta_{k}
$$

Consider $\mathbb{E}\left\{\sum_{i=1}^{N}\left[\delta\left(\tau_{k}, i\right)-p_{i}\right]^{2}\right\}=\sum_{i=1}^{N} p_{i}\left(1-p_{i}\right)$, calculate the difference of $V_{k}$ under $v_{k}=0$, and its mathematical expectation is

$$
\mathbb{E}\left\{\Delta V_{k}\right\}=\bar{\eta}_{k+1}^{T} P \bar{\eta}_{k+1}-\eta_{k}^{T} P \eta_{k},
$$

where $\bar{\eta}_{k+1}=\mathscr{A}_{k} \eta_{k}+\sum_{i=1}^{N} p_{i}\left(1-p_{i}\right) \mathscr{A}_{0} \eta_{k}+\mathscr{E}_{k} g\left(Z \eta_{k}\right)$. Formula (3) can be rewritten as

$$
\left[\begin{array}{c}
x_{k} \\
g\left(x_{k}\right)
\end{array}\right]^{T}\left[\begin{array}{cc}
\mathscr{T}_{1} & \mathscr{T}_{2} \\
\mathscr{T}_{2}^{T} & I
\end{array}\right]^{T}\left[\begin{array}{c}
x_{k} \\
g\left(x_{k}\right)
\end{array}\right] \leq 0 .
$$

By defining $\xi_{k}=\left[\begin{array}{ll}\eta_{k}^{T} & g^{T}\left(Z \eta_{k}\right)\end{array}\right]^{T}$, we can obtain

$$
\mathbb{E}\left\{\Delta V_{k}\right\} \leq \xi_{k}^{T}\left[\begin{array}{cc}
\widetilde{\Phi}_{11} & \widetilde{\Phi}_{12} \\
* & \widetilde{\Phi}_{22}
\end{array}\right] \xi_{k}
$$

where $\widetilde{\Phi}_{11}=\mathscr{A}_{k}^{T} P \mathscr{A}_{k}+\sum_{i=1}^{N} p_{i}\left(1-p_{i}\right) \mathscr{A}_{0}^{T} P \mathscr{A}_{0}-P-\delta \mathscr{T}_{1}$, $\widetilde{\Phi}_{12}=\mathscr{A}_{k}^{T} P \mathscr{E}_{k}-\delta \mathscr{T}_{2}$, and $\widetilde{\Phi}_{22}=\mathscr{E}_{k}^{T} P \mathscr{E}_{k}-\delta I$.

It can be inferred from LMI (14) that

$$
\left[\begin{array}{cc}
\widetilde{\Phi}_{11} & \widetilde{\Phi}_{12} \\
* & \widetilde{\Phi}_{22}
\end{array}\right]+\left[\begin{array}{cc}
\mathscr{C}^{T} \mathscr{C} & 0 \\
0 & 0
\end{array}\right]<0
$$

furthermore we can obtain $\mathbb{E}\left\{\Delta V_{k}\right\}<0$. From the above proof, it can be determined that the fault detection general system (11) is asymptotically mean-square stable [32].

Let us consider the $H_{\infty}$ disturbance restraint conditions. For optional $v_{k} \neq 0$, it can be inferred from (14) and (18) that $\mathbb{E}\left\{\Delta V_{k}\right\}+\mathbb{E}\left\{\widetilde{r}_{k}^{T} \widetilde{r}_{k}\right\}-\gamma^{2} \mathbb{E}\left\{v_{k}^{T} v_{k}\right\}=\mathbb{E}\left\{\widetilde{\xi}_{k}^{T} \Phi \widetilde{\xi}_{k}\right\}<0$, where $\widetilde{\xi}_{k}=$ $\left[\begin{array}{lll}\eta_{k}^{T} & g^{T}\left(Z \eta_{k}\right) & v_{k}^{T}\end{array}\right]^{T}$. Then sum up the inequality from 0 to $\infty$ to obtain

$$
\sum_{k=0}^{\infty} \mathbb{E}\left\{\left|r_{k}\right|^{2}\right\}<\gamma^{2} \sum_{k=0}^{\infty} \mathbb{E}\left\{\left|v_{k}\right|^{2}\right\}-\mathbb{E}\left\{V_{\infty}\right\}+\mathbb{E}\left\{\Delta V_{0}\right\} .
$$

Since the Lyapunov function is positive-definite, it is easy to see that (13) holds under the initial condition. This concludes the proof.

3.2. Robust Fault Detection Filter Design. Before we discuss the robust fault detection filter design problem, the following two lemmas are useful in deriving the filter design results.

Lemma 2 (see $[33,34]$ (Schur complement)). Symmetrical matrix

$$
S=\left[\begin{array}{cc}
S_{11} & S_{12} \\
* & S_{22}
\end{array}\right]
$$

is negative-definite, when and only when

$$
\text { both } S_{11}<0 \text { and } S_{22}-S_{12}^{T} S_{11}^{-1} S_{12}<0 \text {, }
$$

or

$$
\text { both } S_{22}<0 \text { and } S_{11}-S_{12}^{T} S_{22}^{-1} S_{12}<0 \text {. }
$$

Lemma 3 (see [30]). If $M=M^{T}, H$ and $E$ are real matrices, and $F$ satisfies $F_{k} F_{k}^{T} \leq I$, then

$$
M+H F E+E^{T} F^{T} H^{T}<0
$$

holds, when and only when there exists a constant $\varepsilon>0$, making the following LMI holds:

$$
M+\frac{1}{\varepsilon} H H^{T}+\varepsilon E^{T} E<0,
$$

or

$$
\left[\begin{array}{ccc}
M & H & \varepsilon E^{T} \\
* & -\varepsilon I & 0 \\
* & * & -\varepsilon I
\end{array}\right]<0 .
$$

Theorem 4. For a given scalar $\gamma>0$ and the nonlinear discrete time-variant system characterized by (1) (3), there exists a robust fault detection filter in the form of (10), which makes the system asymptotically mean-square stable. And the sufficient condition satisfying the $H_{\infty}$ disturbance restraint conditions is that there exist real matrices $R=R^{T}>0, S=S^{T}>0, U_{1}, U_{2}$, $U_{3}$ and real scalar $\delta, \varepsilon>0$ to make the following LMI holds:

$$
\left[\begin{array}{cccccccccccc}
\Psi_{1,1} & \Psi_{1,2} & -\delta \mathscr{T}_{2} & 0 & 0 & A^{T} S & \Psi_{1,7} & 0 & \Psi_{1,9} & U_{3}^{T} & 0 & \varepsilon H_{1}^{T} \\
* & \Psi_{2,2} & -\delta \mathscr{T}_{2} & 0 & 0 & A^{T} S & \Psi_{2,7} & 0 & \Psi_{2,9} & 0 & 0 & \varepsilon H_{1}^{T} \\
* & * & -\delta I & 0 & 0 & E^{T} S & E^{T} R & 0 & 0 & 0 & 0 & \varepsilon H_{2}^{T} \\
* & * & * & -\gamma^{2} I & 0 & B^{T} S & \Psi_{4,7} & 0 & 0 & 0 & 0 & \varepsilon H_{3}^{T} \\
* & * & * & * & -\gamma^{2} I & M^{T} S & M^{T} R & 0 & 0 & -I & 0 & \varepsilon H_{4}^{T} \\
* & * & * & * & * & -S & -S & 0 & 0 & 0 & N & 0 \\
* & * & * & * & * & * & -R & 0 & 0 & 0 & N & 0 \\
* & * & * & * & * & * & * & -S & -S & 0 & 0 & 0 \\
* & * & * & * & * & * & * & * & -R & 0 & 0 & 0 \\
* & * & * & * & * & * & * & * & * & -I & 0 & 0 \\
* & * & * & * & * & * & * & * & * & * & -\varepsilon I & 0 \\
* & * & * & * & * & * & * & * & * & * & * & -\varepsilon I
\end{array}\right]<0,
$$


where $\Psi_{1,1}=\Psi_{1,2}=-\delta \mathscr{T}_{1}-S, \Psi_{1,7}=A^{T} R+\sum_{i=1}^{N} p_{i}(I+$ $\left.F_{k} \Delta_{i}\right) C^{T} U_{2}^{T}+U_{1}^{T}, \Psi_{1,9}=\Psi_{2,9}=\sigma\left(I+F_{k} \Delta_{i}\right) C^{T} U_{2}^{T}, \Psi_{2,2}=$ $-\delta \mathscr{T}_{1}-R, \Psi_{2,7}=A^{T} R+\sum_{i=1}^{N} p_{i}\left(I+F_{k} \Delta_{i}\right) C^{T} U_{2}^{T}, \Psi_{4,7}=B^{T} R+$ $\sum_{i=1}^{N} p_{i}\left(I+F_{k} \Delta_{i}\right) D^{T} U_{2}^{T}$, and $\sigma=\sum_{i=1}^{N} \sqrt{p_{i}\left(1-p_{i}\right)}$. If LMI (27) holds, then the robust fault detection filter parameters satisfying the above conditions can be calculated by

$$
G=X_{12}^{-1} U_{1} S^{-1} Y_{12}^{-T}, \quad K=X_{12}^{-1} U_{2}, \quad L=U_{3} S^{-1} Y_{12}^{-T},
$$

and $X_{12}$ and $Y_{12}$ here can be determined by $I-R S^{-1}=$ $X_{12} Y_{12}^{T}<0$.

Proof. It can be inferred from Lemmas 2 and 3 that the LMI (14) equals

$$
\left[\begin{array}{cccccccc}
-P-\delta \overline{\mathscr{T}}_{1} & -\delta \overline{\mathscr{T}}_{2} & 0 & \mathscr{A} P & \sigma \mathscr{A}_{0}^{T} P & \mathscr{C}^{T} & 0 & \varepsilon \mathscr{H}_{1}^{T} \\
* & -\delta I & 0 & \mathscr{E} P & 0 & 0 & 0 & \varepsilon H_{2}^{T} \\
* & * & -\gamma^{2} I & \mathscr{B} P & 0 & \mathscr{D}^{T} & 0 & \varepsilon \mathscr{H}_{3}^{T} \\
* & * & * & -P & 0 & 0 & \mathcal{N} & 0 \\
* & * & * & * & -P & 0 & 0 & 0 \\
* & * & * & * & * & -I & 0 & 0 \\
* & * & * & * & * & * & -\varepsilon I & 0 \\
* & * & * & * & * & * & * & -\varepsilon I
\end{array}\right]<0 .
$$

Define $P$ and $P^{-1}$ as the following blocks:

$$
P=\left[\begin{array}{cc}
R & X_{12} \\
X_{12}^{T} & X_{22}
\end{array}\right], \quad P^{-1}=\left[\begin{array}{cc}
S^{-1} & Y_{12} \\
Y_{12}^{T} & Y_{22}
\end{array}\right]
$$

where the dimension of a block is as the same as $\mathscr{A}$.
By defining

$$
J_{1}=\left[\begin{array}{cc}
S^{-1} & I \\
Y_{12}^{T} & 0
\end{array}\right], \quad J_{2}=\left[\begin{array}{cc}
I & R \\
0 & X_{12}^{T}
\end{array}\right]
$$

it can be see that $P J_{1}=J_{2}, J_{1}^{T} P J_{1}=J_{1}^{T} J_{2}$.

By conducting contragradient transformation to the matrix of (29) with $\operatorname{diag}\left\{J_{1}^{T}, I, I, J_{1}^{T}, J_{1}^{T}, I, I, I\right\}$, we can obtain

$$
\left[\begin{array}{cccccccccccc}
\Psi_{1,1} & \Psi_{1,2} & -\delta S^{-T} \mathscr{T}_{2} & 0 & 0 & S^{-T} A^{T} & \Psi_{1,7} & 0 & \Psi_{1,9} & Y_{12} L^{T} & 0 & \varepsilon S^{-T} H_{1}^{T} \\
* & \Psi_{2,2} & -\delta T_{2} & 0 & 0 & A^{T} & \Psi_{2,7} & 0 & \Psi_{2,9} & 0 & 0 & \varepsilon H_{1}^{T} \\
* & * & -\delta I & 0 & 0 & E^{T} & E^{T} R & 0 & 0 & 0 & 0 & \varepsilon H_{2}^{T} \\
* & * & * & -\gamma^{2} I & 0 & B^{T} & \Psi_{4,7} & 0 & 0 & 0 & 0 & \varepsilon H_{3}^{T} \\
* & * & * & * & -\gamma^{2} I & M^{T} & M^{T} R & 0 & 0 & -I & 0 & \varepsilon H_{4}^{T} \\
* & * & * & * & * & -S^{-T} & -I & 0 & 0 & 0 & S^{-T} N & 0 \\
* & * & * & * & * & * & -R & 0 & 0 & 0 & N & 0 \\
* & * & * & * & * & * & * & -S^{-T} & -I & 0 & 0 & 0 \\
* & * & * & * & * & * & * & * & -R & 0 & 0 & 0 \\
* & * & * & * & * & * & * & * & * & -I & 0 & 0 \\
* & * & * & * & * & * & * & * & * & * & -\varepsilon I & 0 \\
* & * & * & * & * & * & * & * & * & * & * & -\varepsilon I
\end{array}\right]<0,
$$

where $\Psi_{1,1}=-\delta S^{-T} \mathscr{T}_{1} S^{-1}-S^{-T}, \Psi_{1,2}=-\delta S^{-T} \mathscr{T}_{1}-I$, $\Psi_{1,7}=S^{-T} A^{T} R+S^{-T} \sum_{i=1}^{N} p_{i}\left(I+F_{k} \Delta_{i}\right) C^{T} K^{T} X_{12}^{T}, \Psi_{1,9}=$ $\sigma S^{-T}\left(I+F_{k} \Delta_{i}\right) C^{T} K^{T} X_{12}^{T}, \Psi_{2,2}=-\delta \mathscr{T}_{1}-R, \Psi_{2,7}=A^{T} R+$ $\sum_{i=1}^{N} p_{i}\left(I+F_{k} \Delta_{i}\right) C^{T} K^{T} X_{12}^{T}, \Psi_{2,9}=\sigma\left(I+F_{k} \Delta_{i}\right) C^{T} K^{T} X_{12}^{T}$, and $\Psi_{4,7}=B^{T} R+\sum_{i=1}^{N} p_{i}\left(I+F_{k} \Delta_{i}\right) D^{T} K^{T} X_{12}^{T}$.
By introducing new matrices $U_{1}=X_{12} G Y_{12}^{T} S, U_{2}=X_{12} K$, and $U_{3}=L Y_{12}^{T} S$ and conducting contragradient transformation to the matrix of (32) with $\operatorname{diag}\{S, I, I, I, I, S, I, S, I, I, I, I\}$, we can obtain LMI (27). It is to say that if the LMI (27) holds, the fault detection augmentation system (11) is ensured to 
be asymptotically mean-square stable and satisfies the $H_{\infty}$ disturbance restraint conditions (13).

On the other hand, if LMI (27) holds, then

$$
\left[\begin{array}{ll}
-S & -S \\
-S & -R
\end{array}\right]<0
$$

and it can be inferred that $S-R<0, S>0$. Since $P P^{-1}=I$, then $I-R S^{-1}=X_{12} Y_{12}^{T}<0$. By matrix singular value decomposing, two nonsingular square matrices $X_{12}$, $Y_{12}$ satisfying conditions can always be obtained; thus the filter parameters can be calculated by (28). This concludes the proof.

3.3. Residual Evaluation Strategy. After the fault detection filter is designed, the residual evaluation function $J_{k}$ and threshold value $J_{\text {th }}$ are introduced to evaluate NSs (whether there are faults in NSs):

$$
\begin{gathered}
J_{k}=\left\{\sum_{s=0}^{k} r_{r}^{T} r_{s}\right\}^{1 / 2}, \\
J_{\text {th }}=\sup _{w \in l_{2}, f=0} \mathbb{E}\left\{J_{k}\right\} .
\end{gathered}
$$

The following inequalities are used to determine whether the fault occurs:

$$
\begin{gathered}
J_{k}>J_{\mathrm{th}} \Longrightarrow \text { Fault } \Longrightarrow \text { ALARM } \\
J_{k} \leq J_{\mathrm{th}} \Longrightarrow \text { No Fault. }
\end{gathered}
$$

\section{Simulation Results}

In this section, in order to show the effectiveness of the proposed method, a discrete-time linear system linearized from a vehicle system is employed with the following parameters:

$$
\begin{aligned}
& A=\left[\begin{array}{ccc}
-0.1 & 0.1 & 0.4 \\
0.3 & 0.2 & -0.1 \\
0.2 & 0.2 & 0.3
\end{array}\right], \quad E=\left[\begin{array}{lll}
0.2 & 0.1 & 0.7 \\
0.1 & 0.2 & 0.4 \\
0.3 & 0.8 & 0.2
\end{array}\right], \\
& B=\left[\begin{array}{c}
0.4 \\
1.0825 \\
4.0687
\end{array}\right], \quad M=\left[\begin{array}{l}
0.2 \\
0.4 \\
0.5
\end{array}\right], \quad N=\left[\begin{array}{l}
0.3 \\
0.1 \\
0.3
\end{array}\right] \\
& H_{1}=\left[\begin{array}{lll}
0.2 & 0.1 & 0.5
\end{array}\right], \quad H_{2}=\left[\begin{array}{lll}
0.3 & 0.1 & 0.2
\end{array}\right] \text {, } \\
& H_{3}=[0.35], \quad H_{4}=[0.2], \\
& C=\left[\begin{array}{lll}
0.2 & 0.1 & 0.5
\end{array}\right], \quad D=[0.3] \text {. }
\end{aligned}
$$

The nonlinear term $g\left(x_{k}\right)$ is defined as $\left[\begin{array}{llll}g_{1}\left(x_{1 k}\right) & 0 & g_{2}\left(x_{2 k}\right)\end{array}\right]^{T}$, where $g_{1}\left(x_{1 k}\right)=0.2 x_{1 k} \times \sin \left(x_{1 k}\right)$ and $g_{2}\left(x_{2 k}\right)=-0.1 x_{2 k} \times$ $\sin \left(x_{2 k}\right)$. Then we can obtain that

$$
T_{1}=\left[\begin{array}{ccc}
0.25 & 0 & 0 \\
0 & 0 & 0 \\
0 & 0 & 0
\end{array}\right], \quad T_{2}=\left[\begin{array}{ccc}
0 & 0 & 0 \\
0 & 0 & 0 \\
0 & 0 & 0.15
\end{array}\right] .
$$

The parameters of logarithmic quantisers are set as $u_{10}^{(1)}=$ $u_{10}^{(2)}=u_{10}^{(3)}=u_{20}^{(1)}=u_{20}^{(2)}=u_{20}^{(3)}=u_{30}^{(1)}=u_{30}^{(2)}=u_{30}^{(3)}=1$,

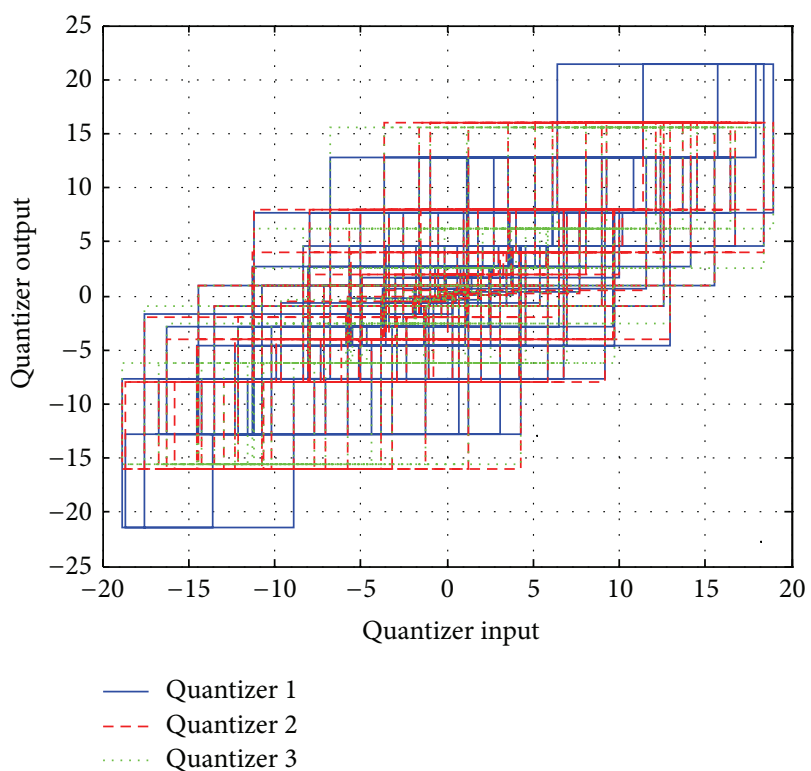

Figure 1: Quantisation law of three quantisers.

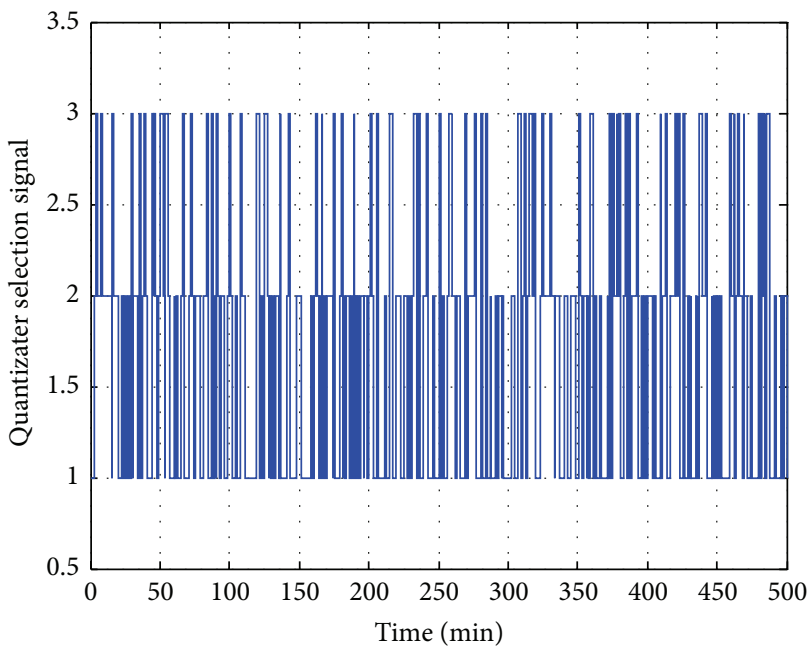

FIGURE 2: Quantiser selection indicator.

$\rho_{1}^{(1)}=\rho_{1}^{(2)}=\rho_{1}^{(3)}=0.6, \rho_{2}^{(1)}=\rho_{2}^{(2)}=\rho_{2}^{(3)}=0.5$, and $\rho_{3}^{(1)}=$ $\rho_{3}^{(2)}=\rho_{3}^{(3)}=0.4$, as shown in Figure 1. The distribution law of the quantiser selection variable $\tau_{k}$ is set as $\operatorname{Prob}\left\{\tau_{k}=1\right\}=0.5$, $\operatorname{Prob}\left\{\tau_{k}=2\right\}=0.35$, and $\operatorname{Prob}\left\{\tau_{k}=3\right\}=0.15$. It is to say that, at a certain time, the probability of quantiser 1 is 0.4 , the probability of quantiser 2 is 0.35 , and the probability of quantiser 3 is 0.25 , which are illustrated in Figure 2.

This paper aims to determine the parameters of the $H_{\infty}$ robust fault detection filter by using Theorem 4 . With the help of mincx in Matlab [35], we can calculate the minimum value of $\gamma$ as $\gamma^{*}=\sqrt{\gamma_{\mathrm{opt}}^{2}}=3.3321$, where $\gamma_{\mathrm{opt}}^{2}$ is the optimal solution of the corresponding convex optimisation problems. 

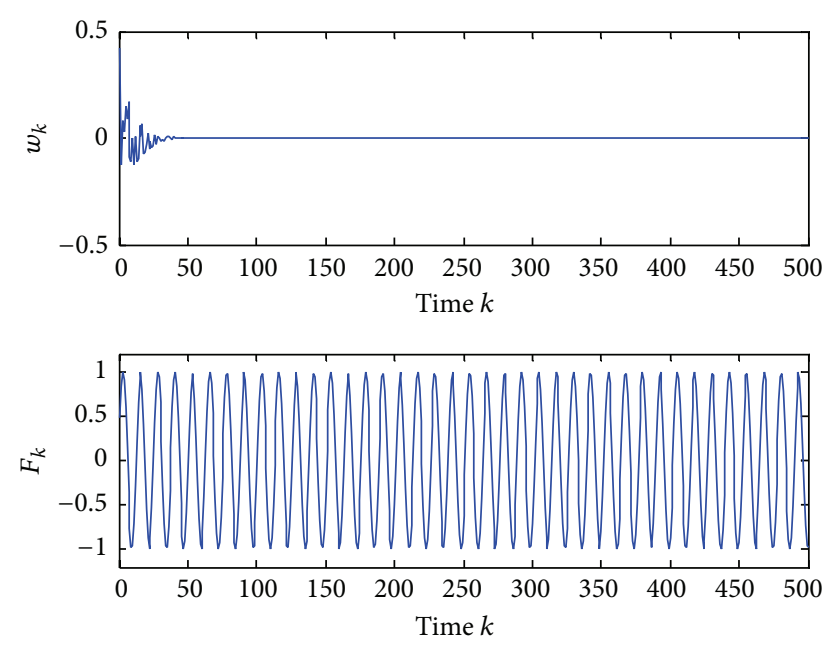

FIgURE 3: The time-domain simulations of $w_{k}$ and $F_{k}$.

The parameters of the $H_{\infty}$ robust fault detection filter are calculated as

$$
\begin{gathered}
G=\left[\begin{array}{ccc}
-0.1985 & -0.2718 & -0.856 \\
0.0005 & 0.3711 & 0.7378 \\
-0.0113 & 0.1834 & 0.6084
\end{array}\right], \quad K=\left[\begin{array}{c}
0.044 \\
-0.1722 \\
-0.031
\end{array}\right], \\
L=\left[\begin{array}{lll}
-0.029 & -0.0192 & 0.0945
\end{array}\right]
\end{gathered}
$$

The unknown input $w_{k}$ is defined as $0.5 \times \exp (-k / 10) \times$ $\left(2 n_{k}-1\right)$, for $k=0,1, \ldots, 500$, where $n_{k}$ is uniformly distributed in $[-1,1]$. The system parameter $F_{k}$ is taken as $\sin (k / 2)$, as illustrated in Figure 3. The fault signal $f_{k}$ is taken as 0.5 when $k \in[200,300]$; otherwise $f_{k}=0$.

As shown in Figure 4, (a) represents system's fault signal; (b) represents the square of residuals $r_{k}$; (c) represents the output of residuals evaluation function $J_{k}$. By fixing the form of $w_{k}$, we obtain $J_{\text {th }}=(1 / 500) \sum_{t=1}^{500} J_{500}=1.5997 \times 10^{-4}$ after 500 times of simulation without fault. As demonstrated in Figure 4(c), residuals evaluation function $J_{260}=1.5975 \times$ $10^{-4}<J_{\text {th }}<J_{261}=1.6462 \times 10^{-4}$; then it can be seen that the fault is detected in the 61st step after it occurred.

\section{Conclusion}

In this paper, the analysis and design problem of an $H_{\infty}$ robust fault detection filter for a class of discrete-time NSs with ROQs have been investigated. Because the bandwidth of the communication channel is limited, signal must be quantised before it is transmitted via network. ROQs are introduced in the form of randomly occurring logarithmic quantisers, which are transformed into randomly occurring uncertainties of system parameters. By vector augmenting, the original fault detection problem is transformed into robust $H_{\infty}$ filter problem. Then a sufficient condition is presented to ensure the existence of robust fault detection filter, which guarantees the asymptotically mean-square stability of fault detection system and satisfies a certain $H_{\infty}$ disturbance

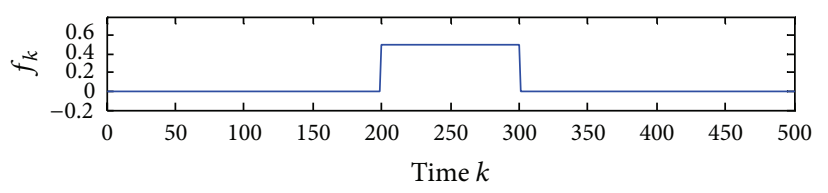

(a)

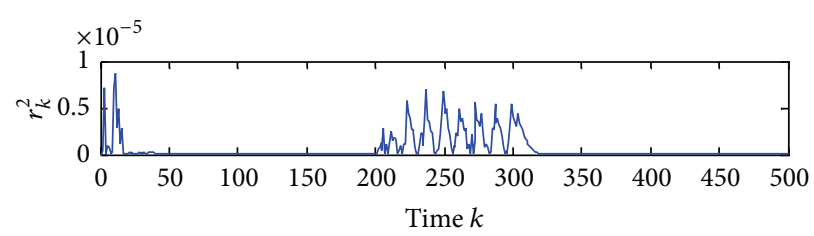

(b)

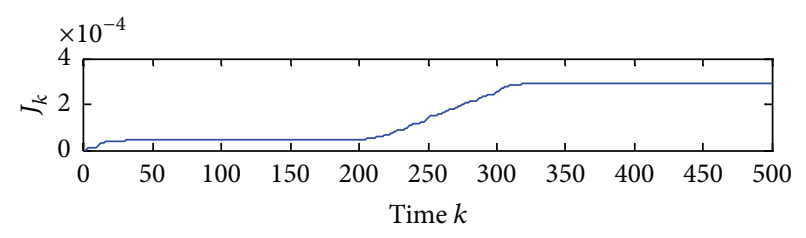

(c)

FIgURE 4: The time-domain simulations of $f_{k}, r_{k}^{2}$ and $J_{k}$.

restraint condition. The simulation results demonstrate that this proposed approach is efficient for NSs with ROQs.

\section{Conflict of Interests}

The authors declare that there is no conflict of interests regarding the publication of this paper.

\section{References}

[1] W. Zhang, M. S. Branicky, and S. M. Phillips, "Stability of networked control systems," IEEE Control Systems Magazine, vol. 21, no. 1, pp. 84-99, 2001.

[2] M.-Y. Chow and Y. Tipsuwan, "Network-based control systems: a tutorial," in Proceedings of the 27th Annual Conference of the IEEE Industrial Electronics Society (IECON '01), pp. 1593-1602, Denver, Colo, USA, December 2001.

[3] J. P. Hespanha, P. Naghshtabrizi, and Y. Xu, "A survey of recent results in networked control systems," Proceedings of the IEEE, vol. 95, no. 1, pp. 138-172, 2007.

[4] L. G. Bushnell, "Special issue on networks and control," IEEE Control Systems Magazine, vol. 21, no. 1, pp. 132-143, 2001.

[5] P. Antsaklis and J. Baillieul, "Special issue on networked control systems," IEEE Transactions on Automatic Control, vol. 49, no. 4, pp. 35-46, 2004.

[6] F.-Y. Wang, D. Liu, S. X. Yang, and L. Li, "Networking, sensing, and control for networked control systems: architectures, algorithms, and applications," IEEE Transactions on Systems, Man and Cybernetics. Part C: Applications and Reviews, vol. 37, no. 2, pp. 157-159, 2007.

[7] T. J. Koo and S. Sastry, "Special issue on networked embedded hybrid control systems," Asian Journal of Control, vol. 10, no. 1, pp. 103-115, 2008. 
[8] R. M. Murray, K. M. Astrom, S. P. Boyd, R. W. Brockett, and G. Stein, "Future directions in control in an information-rich world," IEEE Control Systems, vol. 23, no. 2, pp. 20-33, 2003.

[9] D. Liberzon, "On stabilization of linear systems with limited information," IEEE Transactions on Automatic Control, vol. 48, no. 2, pp. 304-307, 2003.

[10] S. Wlmadssia, K. Saadaoui, and M. Benrejeb, "New delaydependent stability conditions for linear systems with delay," System Science and Control Engineering, vol. 1, no. 1, pp. 2-11, 2013.

[11] L. Zhang, Y. Shi, T. Chen, and B. Huang, "A new method for stabilization of networked control systems with random delays," IEEE Transactions on Automatic Control, vol. 50, no. 8, pp. 11771181, 2005.

[12] D. Yue, Q.-L. Han, and J. Lam, "Network-based robust $H_{\infty}$ control of systems with uncertainty," Automatica, vol. 41, no. 6, pp. 999-1007, 2005.

[13] F. Yang, Z. Wang, Y. S. Hung, and M. Gani, “ $H_{\infty}$ control for networked systems with random communication delays," IEEE Transactions on Automatic Control, vol. 51, no. 3, pp. 511-518, 2006.

[14] H. Ahmada and T. Namerikawa, "Extended Kalman, filterbased mobile robot localization with intermittent measurements," System Science and Control Engineering, vol. 1, no. 1, pp. 113-126, 2013.

[15] M. Sahebsara, T. Chen, and S. L. Shah, "Optimal $H_{2}$ filtering in networked control systems with multiple packet dropout," IEEE Transactions on Automatic Control, vol. 52, no. 8, pp. 1508-1513, 2007.

[16] H. J. Gao, Y. Zhao, J. Lam et al., " $H_{¥}$ fuzzy filtering of nonlinear systems with intermittent measurements," IEEE Transactions on Systems Fuzzy Systems, vol. 17, no. 2, pp. 291-300, 2009.

[17] J. Chen and R. J. Patton, Robust Model-Based Fault Diagnosis for Dynamic Systems, Kluwer Academic, Boston, Mass, USA, 1999.

[18] X. S. Ding, T. Jeinsch, M. P. Frank et al., "A unified approach to the optimization of fault detection systems," International Journal of Adaptive Control and Signal Processing, vol. 14, no. 7, pp. 725-745, 2000.

[19] L. Qin, X. He, and D. H. Zhou, "A survey of fault diagnosis for swarm systems," Systems Science \& Control Engineering: An Open Access Journal, vol. 2, no. 1, pp. 13-23, 2014.

[20] H. Fang, H. Ye, and M. Zhong, "Fault diagnosis of networked control systems," Annual Reviews in Control, vol. 31, no. 1, pp. 55-68, 2007.

[21] Y.-Q. Wang, H. Ye, and G.-Z. Wang, "Recent development of fault detection techniques for networked control systems," Control Theory and Applications, vol. 26, no. 4, pp. 400-409, 2009 (Chinese).

[22] X. He, Z. Wang, and D. H. Zhou, "Robust fault detection for networked systems with communication delay and data missing," Automatica, vol. 45, no. 11, pp. 2634-2639, 2009.

[23] W. S. Wong and R. W. Brockett, "Systems with finite communication bandwidth constraints. II. Stabilization with limited information feedback," IEEE Transactions on Automatic Control, vol. 44, no. 5, pp. 1049-1053, 1999.

[24] X. He, Z. Wang, X. Wang, and D. H. Zhou, "Networked strong tracking filtering with multiple packet dropouts: algorithms and applications," IEEE Transactions on Industrial Electronics, vol. 61, no. 3, pp. 1454-1463, 2014.

[25] R. W. Brockett and D. Liberzon, "Quantized feedback stabilization of linear systems," IEEE Transactions on Automatic Control, vol. 45, no. 7, pp. 1279-1289, 2000.
[26] S. Tatikonda and S. Mitter, "Control under communication constraints," IEEE Transactions on Automatic Control, vol. 49, no. 7, pp. 1056-1068, 2004.

[27] X. He, Z. Wang, Y. Liu, and D. H. Zhou, "Least-squares fault detection and diagnosis for networked sensing systems using a direct state estimation approach," IEEE Transactions on Industrial Informatics, vol. 9, no. 3, pp. 1670-1679, 2013.

[28] M. Fu and L. Xie, "The sector bound approach to quantized feedback control," IEEE Transactions on Automatic Control, vol. 50, no. 11, pp. 1698-1711, 2005.

[29] Y. H. Yang, W. Wang, and F. W. Yang, "Quantified $H_{\infty}$ filter for discrete-time MIMO systems," Control and Decision, vol. 24, no. 6, pp. 932-936, 2009 (Chinese).

[30] W. Li, P. Zhang, S. X. Ding, and O. Bredtmann, "Fault detection over noisy wireless channels," in Proceedings of the 46th IEEE Conference on Decision and Control (CDC '07), pp. 5050-5055, New Orleans, La, USA, December 2007.

[31] Q.-L. Han, "Absolute stability of time-delay systems with sectorbounded nonlinearity," Automatica, vol. 41, no. 12, pp. 21712176, 2005.

[32] X. Mao, Differential Equations and Applications, Horwood Publishing, Chichester, UK, 1997.

[33] S. Boyd, L. El Ghaoui, E. Feron, and V. Balakrishnan, Linear Matrix Inequalities in System and Control Theory, SIAM, Philadelphia, Pa, USA, 1994.

[34] K. M. Grigoriadis and J. T. Watson Jr., "Reduced-order $H_{\infty}$ and $L_{2}-L_{\infty}$ filtering via linear matrix inequalities," IEEE Transactions on Aerospace and Electronic Systems, vol. 33, no. 4, pp. 1326-1338, 1997.

[35] P. Gahinet, A. Nemirovski, J. A. Laub et al., LMI Control Toolbox-for Use with Matlab, The Math Works, Natick, Mass, USA, 1995. 


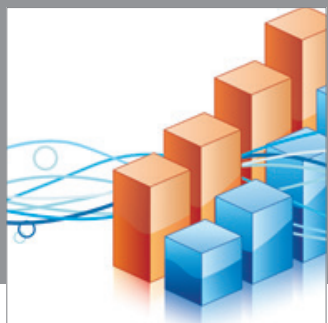

Advances in

Operations Research

mansans

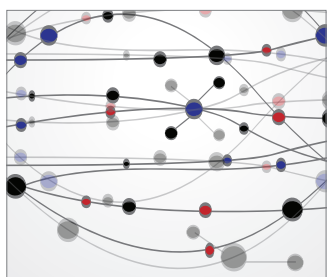

The Scientific World Journal
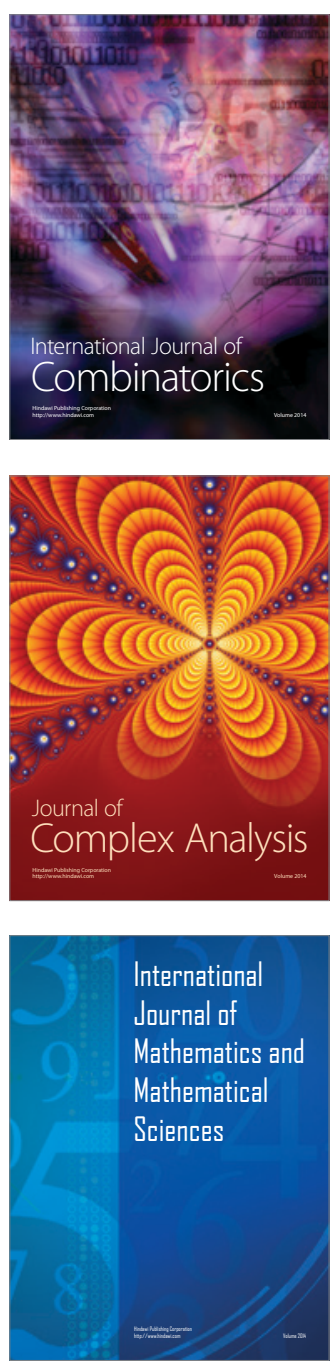
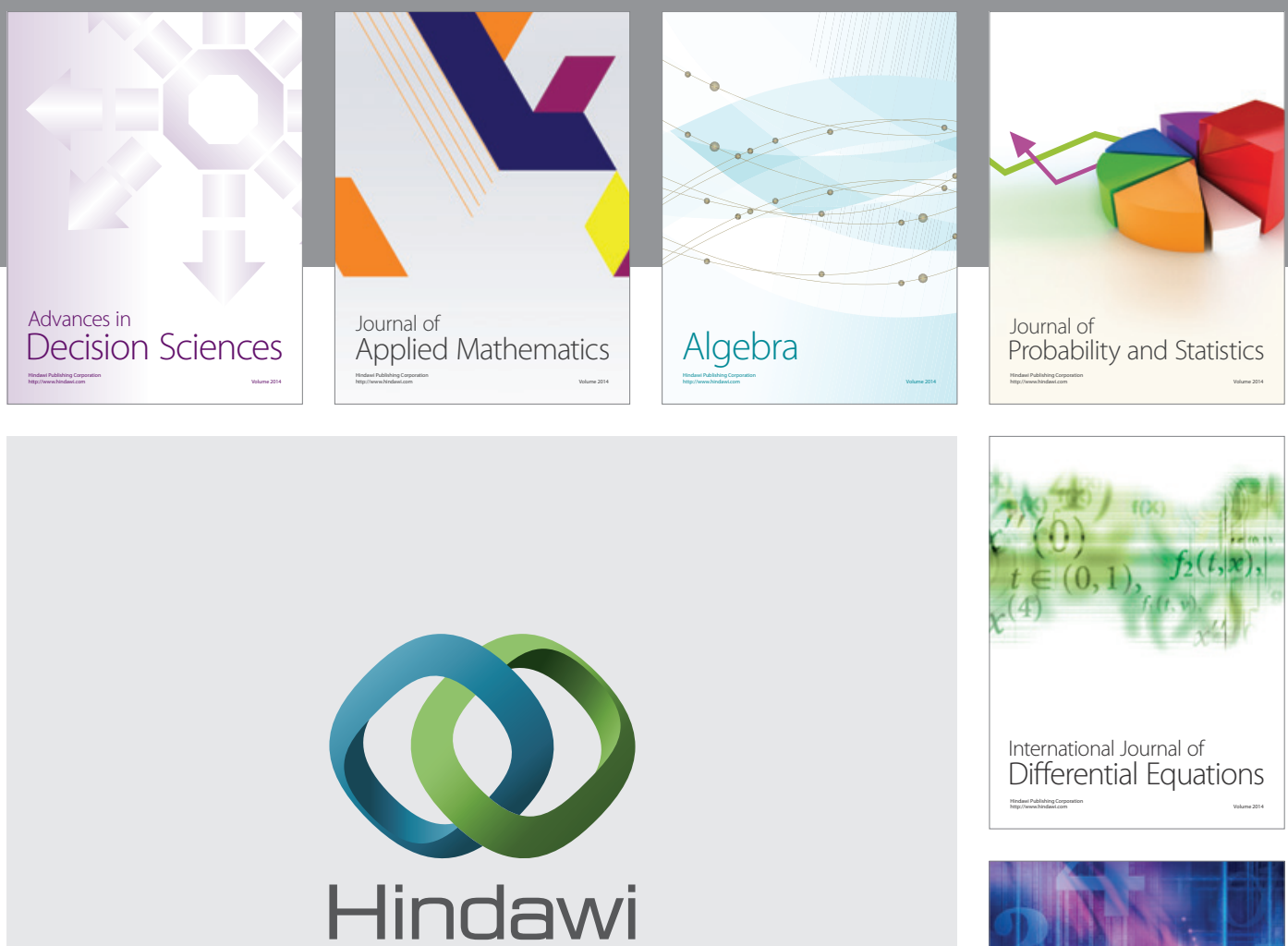

Submit your manuscripts at http://www.hindawi.com
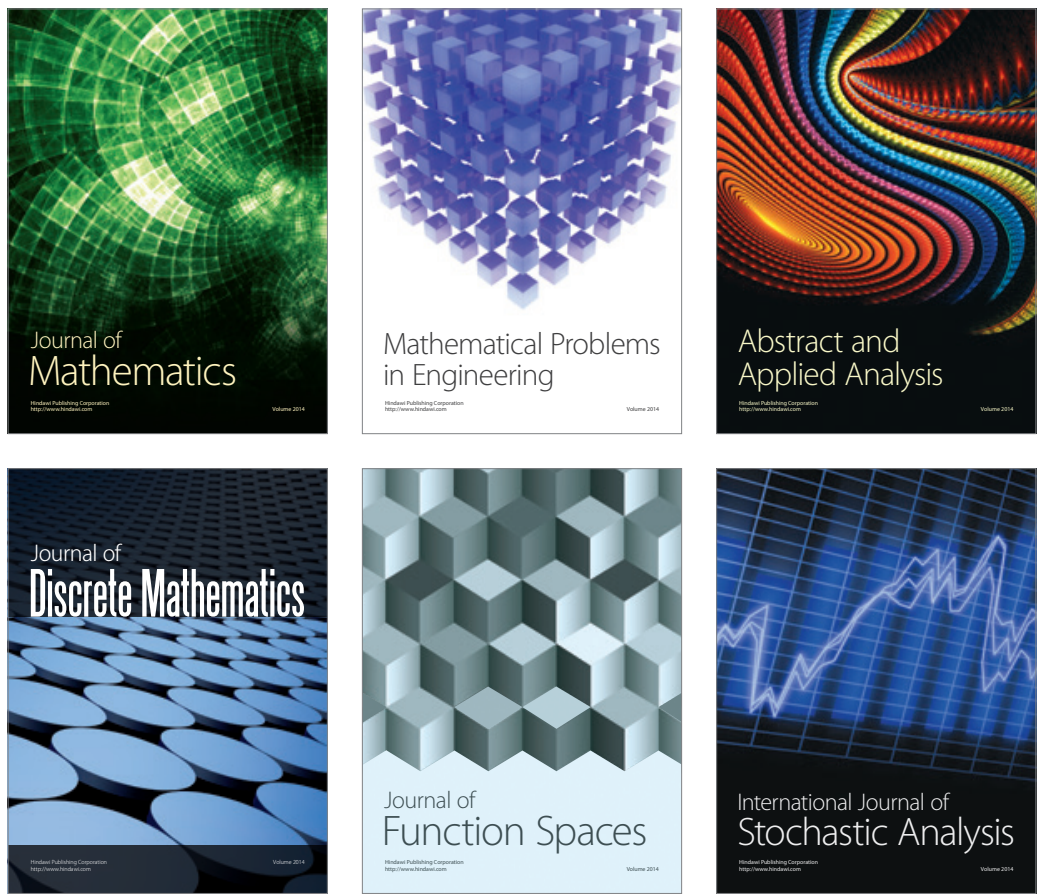

Journal of

Function Spaces

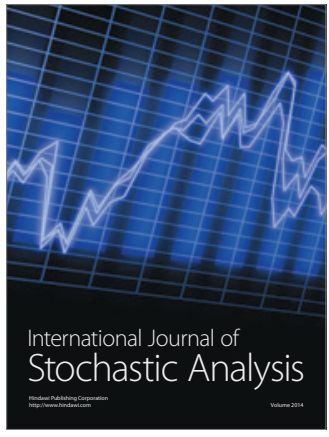

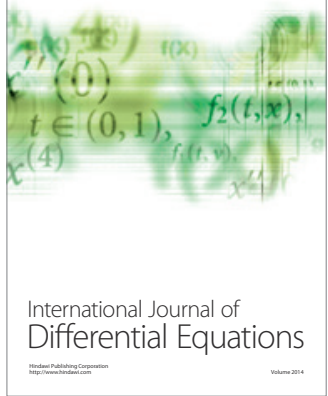
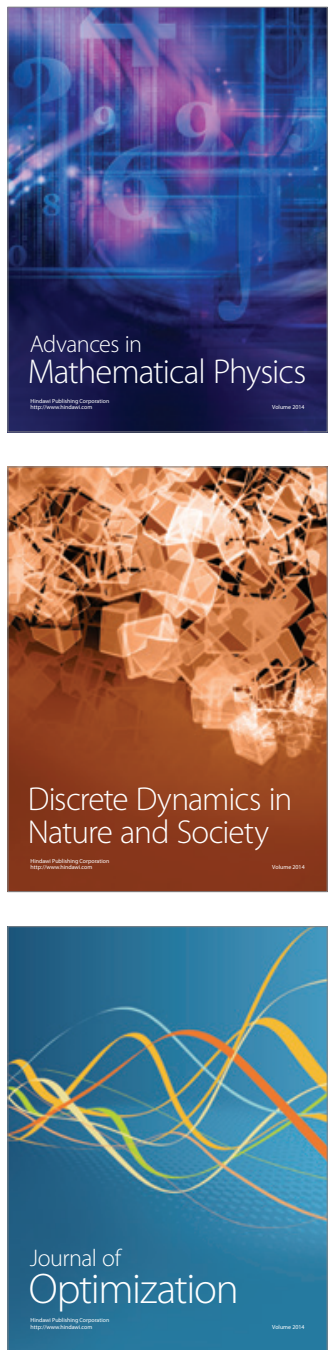